\title{
Efecto de tubos protectores en el crecimiento inicial de plántulas de Tetrorchidium rubrivenium en Huancabamba, Oxapampa, Perú
}

\author{
Effect of tree shelters on the initial growth of Tetrorchidium rubrivenium seedlings \\ in Huancabamba, Oxapampa, Peru
}

Luis M. Llerena Bermúdez¹, Antonio D. Del Campo García², Ignacio R. Lombardi Indacochea ${ }^{3}$, Carlos A. Llerena Pinto ${ }^{3}$ y Consuelo Arellano Ugarte ${ }^{4}$

\begin{abstract}
Resumen
Este estudio se llevó a cabo para evaluar los posibles efectos del tubo protector o tree shelter, en el establecimiento y crecimiento inicial de plantones de Tetrorchidium rubrivenium, "col de monte" (su influencia en el crecimiento en altura y diámetro, y el aporte hídrico por condensación de neblinas en sus paredes internas). Se realizó en el distrito de Huancabamba, provincia de Oxapampa, Pasco y consistió de una plantación de 120 individuos en una parcela ubicada en el Fundo Ranchería ( $\mathrm{km} \mathrm{1.1,} \mathrm{carretera}$ de Chorobamba). Los tratamientos fueron 5 diámetros de tubos: D1-8,65 cm, D2-9,8 $\mathrm{cm}, \mathrm{D} 3-10,5 \mathrm{~cm}, \mathrm{D} 4-11,5 \mathrm{~cm}, \mathrm{D} 5-12,15 \mathrm{~cm}$ y un tratamiento testigo; además de tener tubos con ventilación y sin ventilación, $50 \%$ en cada tipo, lo cual arroja un total de 11 variables en estudio: 10 con tubos protectores ( 5 diámetros de tubos x 2 tipos de tubos) y 1 testigo. Los resultados de supervivencia con los tubos protectores fueron muy bajos, siendo $50 \%$ el máximo alcanzado por el tubo de $10,5 \mathrm{~cm}$, a diferencia del $90 \%$ alcanzado por el testigo. El crecimiento en altura fue mucho mayor dentro de los tubos debido a la ausencia de luz solar que genera un rápido crecimiento vertical (etiolación), con disminución de la tasa de crecimiento en diámetro. En los testigos se dio la situación contraria, mayor crecimiento en diámetro pero plantas más bajas. La humedad del suelo fue mayor en plantas con tubos por el aporte extra de agua de las neblinas pero no fue suficiente para disminuir el efecto de las altas temperaturas generadas dentro de los tubos que causó la alta mortalidad, aquí la ventilación tuvo un efecto primordial ya que comparando tubos ventilados con no ventilados, los últimos mostraron un menor porcentaje de supervivencia, $22 \%$ a diferencia del 54\% de los tubos ventilados.
\end{abstract}

Palabras clave: supervivencia; altura; diámetro; establecimiento de plantas; tubo protector.

\footnotetext{
${ }^{1}$ Consultor independiente, Perú; e-mail: luisllb@yahoo.com

${ }^{2}$ Universidad Politécnica de Valencia, España.

${ }^{3}$ Facultad de Ciencias Forestales, Universidad Nacional Agraria La Molina, Perú.

${ }^{4}$ Departamento de Estadística, North Carolina State University, EE.UU.
} 


\begin{abstract}
This study was carried out to evaluate the possible effects of the tree shelters in the initial establishment and growth of Tetrorchidium rubrivenium seedlings, its influence on diameter and height growth, and hydric contribution by mist condensation on its inner walls. It was performed at the district of Huancambamba, Oxapampa, province, Pasco. The evaluation consisted in a plantation of 120 individuals in a plot located in the "Rancheria" farm (Chorobamba road, km 1.1). The treatments were 5 shelter diameters: D1-8,65 cm; D2-9,8 cm; D3-10,5 cm; D4-11,5 cm; D5-12,15 cm and a control treatment without shelter, in addition there are shelters with and without ventilation, $50 \%$ each, which yields a total of 11 variables under study: 10 with tree shelters (5 different diameters $\mathrm{x} 2$ types) and 1 control. The survival results with tree shelters were very low, being $50 \%$ the maximum reached by the $10,5 \mathrm{~cm}$ shelter unlike the $90 \%$ achieved by the control. The height growth was much better inside the shelter due to the absence of light that generates a faster vertical growth (etiolation), but a decrease in the diameter rate growth. The situation was opposite with the control treatment, more diameter growth but smaller plants. The soil moisture was higher inside the shelters but not enough to reduce the mortality because of the high temperatures inside the shelters, in this point the ventilation had and important effect in the survival variable, $54 \%$ with ventilated shelter as opposed to $22 \%$ with non-ventilated shelters.
\end{abstract}

Key words: survival; height; diameter; plants establishment; tree shelter.

\section{Introducción}

Uno de los principales parámetros que influyen en el establecimiento exitoso de programas de reforestación y en el desarrollo de las plantaciones forestales es el agua, recurso vital que se encuentra en diferentes formas y estados dependiendo de la ubicación y las condiciones del sitio en donde se pretende establecer una plantación. Una de las formas naturales del agua es la niebla o neblina, la cual ha sido poco estudiada ya que es una variable climática difícil de analizar por la ausencia de instrumentos específicos que la midan con precisión, además de su presencia aleatoria (Marzol 2005). De acuerdo a Acosta (1996) la Organización Meteorológica Mundial (OMM), define como niebla a una suspensión en el aire de gotas muy pequeñas de agua, habitualmente microscópicas, que generalmente reducen la visibilidad horizontal en la superficie de la tierra, a menos de un kilómetro. Este recurso, adecuadamente aprovechado, podría representar una alternativa ecológica y económicamente importante de agua en zonas en donde no existan otras fuentes accesibles, y permitir el desarrollo de algunos cultivos agrícolas, forestales o agroforestales o aportes de agua para otros fines (Acosta 1996, Marzol 2005).

La zona donde se realiza este estudio, distrito de Huancabamba, Provincia de Oxapampa, Región Pasco, se caracteriza por presentar una precipitación total anual mayor a los 2000 $\mathrm{mm}$ que se concentra entre los meses de octubre y abril (Catchpole 2012), siendo el inicio de esta estación lluviosa el momento idóneo para las plantaciones forestales. No obstante, la ejecución de las plantaciones en la época de menor pluviometría puede favorecer, además de las condiciones de trabajo y con ello el rendimiento, el crecimiento temprano de los plantones frente a la maleza competitiva, que se desarrolla sobre todo a partir del comienzo de las lluvias. La competencia de esta maleza llega a ser uno de los principales problemas para el establecimiento de plantaciones en los trópicos (Gutierrez 2007). Entre mayo y septiembre cuando las lluvias disminuyen, las neblinas representan un aporte adicional importante de agua para la vegetación. Sin embargo, la ausencia de una pantalla vegetal interceptora puede 
suponer la pérdida de estas cantidades de agua y con ello crear estrés hídrico en plantaciones forestales, que habitualmente son realizadas en este tipo de terrenos deforestados. En este sentido, el presente trabajo busca probar una tecnología simple y práctica que mejore las condiciones hídricas del sitio en donde se instala una plántula en el terreno definitivo durante la época seca, a través del aprovechamiento del agua atmosférica de las nieblas bajas, utilizando tubos protectores. Estos tubos de material plástico ligero, laminado y corrugado, son colocados en forma vertical y estable alrededor de los árboles plantados, actúan como eficientes "atrapa nieblas" por su amplia superficie de captación de la niebla. Además debido a su mayor capacidad de enfriamiento durante la noche que su entorno inmediato, pueden colectar cantidades apreciables de rocío que se depositan en el suelo por goteo (del Campo et al. 2006).

Los tubos pueden además, generar un microclima favorable a la planta, proteger su integridad ante posibles amenazas climáticas (vientos o heladas), de la fauna silvestre o doméstica, así como de la competencia vegetal de especies locales agresivas (Kjelgren y Rupp 1997, Kjelgren et al. 1997). Esta tecnología muy usada en Europa en trabajos de reforestación, tiene la ventaja que no implica grandes inversiones adicionales, no requiere equipos sofisticados y genera poco impacto local ya que no requiere instalaciones especiales más allá del tubo mismo. Además, los tubos no dañan al medio ambiente y están estabilizados contra radiación UV, de acuerdo a las especificaciones de la empresa fabricante (Tubex 2006).

Los tubos protectores se conocen en inglés como tree shelters y fueron creados en Inglaterra en 1979 por Graham Tuley, son tubos translúcidos u opacos que se colocan alrededor de la plántula creando un microclima mientras ofrecen protección contra animales y el propio medio (Potter 1991). Los tubos protectores están usualmente fijados en el suelo con estacas y proveen soporte después de la plantación, disminuyendo los periodos de establecimiento o prendimiento e incrementando las tasas de supervivencia.
El correcto uso de los tubos puede reducir las pérdidas causadas por animales, los costos de aplicación por herbicidas, el tiempo utilizado en inspección y mantenimiento y el estrés asociado con el transporte de las plantas del vivero al campo definitivo. Todo lo indicado implica menores costos de plantación (Tubex 2006). No obstante, se han indicado también algunos efectos negativos sobre la fotosíntesis como consecuencia del agotamiento del $\mathrm{CO}_{2}$ en el interior del tubo, precisamente por las favorables condiciones para el crecimiento de las plantas. Esto se corrige perforando orificios de ventilación en los tubos para provocar corrientes de aire que renueven el $\mathrm{CO}_{2}$ (Bergez y Dupraz 2000). Los tubos protectores imitan las condiciones naturales (bajos niveles de intensidad de luz, elevada humedad y reducidos movimientos de aire) a las que está expuesta una planta en su etapa inicial de crecimiento en un bosque, creando un ambiente caluroso y húmedo en su interior. El efecto neto de los tubos de protección es disminuir las corrientes de aire que causan la pérdida de agua en las plantas manteniendo la humedad. Cuando los tubos se usan en ambientes de humedad atmosférica alta y constante, como los bosques nublados de montaña, pueden incluso captar esta humedad del aire o niebla, condensarla en la superficie y los pliegues del tubo plástico y aportar agua adicional a las plántulas por medio de su escurrimiento al suelo (Tubex 2006).

En base a lo mencionado, se busca medir la eficacia del uso de tubos protectores para plantaciones forestales a través del registro de parámetros de establecimiento inicial como supervivencia, crecimiento en altura y diámetro, y determinar su influencia en el aporte hídrico con valores relativos de humedad del suelo.

\section{Materiales y Métodos}

\section{Material vegetal}

Se utilizaron plantones de la especie forestal nativa "col de monte" (Tetrorchidium rubrivenium, familia Euphorbiaceae) procedentes del vivero forestal de alta tecnología de Fondebosque ubicado en la ciudad de Oxapampa 
(Figura 1). El sustrato que se ha utilizado en el vivero para producir esta especie está compuesto en un $50 \%$ de tierra vegetal y arena, y el otro $50 \%$ con fibra de coco, cascarilla de arroz o vermiculita para mantener la humedad. No se usa una alta proporción de tierra vegetal para reducir la competencia. Los plantones del vivero de Fondebosque provienen de rodales semilleros ubicados y georreferenciados en un radio máximo equivalente a la distancia de Oxapampa con la ciudad de Villa Rica, estos rodales están clasificados de acuerdo a los calendarios fenológicos de las especies que las componen. Una vez obtenidas las semillas se realiza un mejoramiento genético combinando los mejores individuos para comenzar a producirlas en grandes cantidades y obtener una buena trazabilidad que es el objetivo principal de los viveros de alta tecnología. El periodo de vivero de la col de monte es de 6 meses (Filomeno 2008).

Tetrorchidium rubrivenium es una especie de porte mediano a grande, su crecimiento en diámetro está en el rango de $25-70 \mathrm{~cm}$ y en altura 12-30 m, tiene un fuste cilíndrico y las ramificaciones empiezan a partir del segundo tercio, las hojas son simples, alternas y dispuestas en espiral. Las láminas pueden ser elípticas u oblongas y tienen el borde dentado (Reynel et al. 2006).

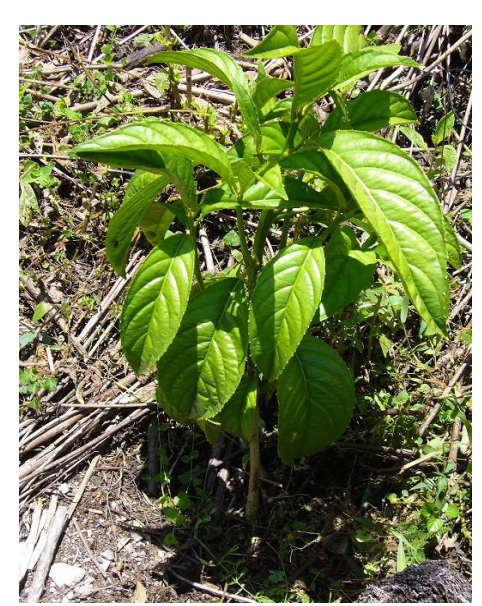

Figura 1. Plántula de Tetrorchidium

rubrivenium.

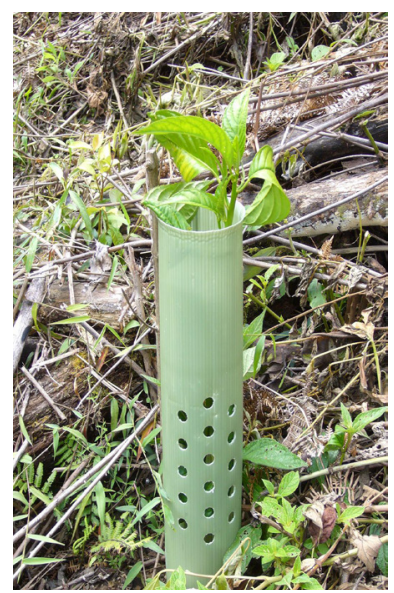

Figura 2. Vista lateral del Tree shelter.

\section{Herramientas y equipos de campo}

\section{- Terreno}

La limpieza del terreno se realizó manualmente con machetes y palas; la demarcación de la parcela con estacas, cinta métrica de $30 \mathrm{~m}$ y un telémetro Bushnell; en la plantación se usó cinta métrica para el espaciamiento y herramientas agrícolas para los hoyos y ubicación de las plántulas. La pendiente del terreno fue medida con un clinómetro (Suunto).

\section{- Mediciones de diámetro y altura}

Las mediciones de diámetros y alturas de las plántulas se realizaron con un calibrador vernier simple y una cinta métrica.

\section{Datos meteorológicos}

Para el registro de datos meteorológicos se instaló un pluviógrafo (Rainwise, que registra lluvias con fecha y hora, por un sistema de topes, cada tope representa $1 \mathrm{~mm}$ ) y un sensor de temperatura (Hobo), con un acumulador de datos (Hobo).

\section{- Humedad del suelo}

Para el control de la humedad del suelo se usó el método de la conductividad eléctrica por medio de bloques de yeso con dos electrodos, cuya resistencia eléctrica se registró con un ohmnímetro (Delmhosrt) con una escala de

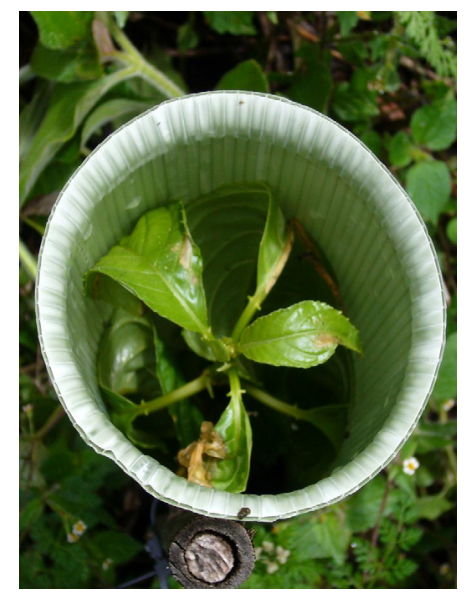

Figura 3. Vista superior del Tree shelter. 
0 a 9 que permite establecer valores relativos con el fin de comparar la humedad entre los diferentes tratamientos. Esté método se basa en el hecho de que la conductividad eléctrica de muchos materiales varían en función de su contenido de humedad (Pearcy et al. 1994).

\section{- Tubos protectores (tree shelters)}

Los tubos protectores utilizados para el presente estudio corresponden a los modelos "Standard treeshelter" y "Standard Plus treeshelter", de $60 \mathrm{~cm}$ de altura, 5 diámetros: D1-8,65 $\mathrm{cm}, \mathrm{D} 2-9,8 \mathrm{~cm}, \mathrm{D} 3-10,5 \mathrm{~cm}, \mathrm{D} 4-11,5 \mathrm{~cm}, \mathrm{D} 5-$ $12,15 \mathrm{~cm}$, de color verde, con y sin agujeros de ventilación, y sellos de plástico para sujeción con estacas (Figuras 2 y 3) (Tubex Standard, 2007).

\section{Tratamientos aplicados}

Un tratamiento corresponde a un tubo protector de un determinado diámetro, presentando o no agujeros de ventilación, más un tratamiento control (testigo) que no presenta un tubo protector.

Para lograr los objetivos del estudio se plantaron 120 individuos. En total se necesitaron 100 tubos, 50 de cada tipo (ventilado y no ventilado). Esto llevó a utilizar 20 plantones por diámetro de tubo (Cuadro 1).

\section{Parámetros considerados y duración del ex- perimento}

Para evaluar el efecto de los tubos se realizaron mediciones de las alturas y diámetros en la base del tallo de las plántulas y se controlaron variables ambientales en base a registros de temperatura (cada 29 minutos) y precipitación

\begin{tabular}{|c|c|c|}
\hline \multirow{2}{*}{$\begin{array}{c}\text { Diámetro de } \\
\text { tubo }(\mathbf{c m})\end{array}$} & \multicolumn{2}{|c|}{ Ventilación } \\
\cline { 2 - 3 } & No Ventilado (NV) & Ventilado (V) \\
\hline 8,65 & 10 & 10 \\
\hline 9,8 & 10 & 10 \\
\hline 10,5 & 10 & 10 \\
\hline 11,5 & 10 & 10 \\
\hline 12,15 & 10 & 10 \\
\hline Testigo & \multicolumn{2}{|c|}{20} \\
\hline
\end{tabular}

Cuadro 1. Tratamientos aplicados. (pluviógrafo). Se tomaron datos relativos de la humedad del suelo por medio de 30 bloques de yeso, 4 por cada diámetro de tubo y 10 para el testigo repartidos en los cinco bloques, cada bloque de yeso ubicado a $5 \mathrm{~cm}$ del tubo o de la planta.

La duración total del ensayo fue de seis (6) meses: del 18 de mayo al 08 de noviembre del 2006, fecha en que se realizó la última medición y se retiraron los tubos protectores. Se hicieron mediciones cada 3 semanas, llevándose a cabo un total de 8 mediciones.

\section{Análisis de datos}

Los datos generados en el estudio fueron procesados y tratados mediante una hoja de cálculo (Excel) y el paquete estadístico SAS. Para la determinación del efecto de los tratamientos sobre el establecimiento, se realizó un análisis estadístico descriptivo según el grado de modificación ambiental inducido (testigo, tubo ventilado y tubo sin ventilar) y el diámetro del tubo protector. Además, se han desarrollado pruebas de t para comparar los resultados entre tratamientos y en relación al testigo.

Como medida de protección contra el Error Tipo I (concluir que el tipo de tubo tiene un efecto significativo sobre la variable respuesta cuando en realidad sus efectos no son distinguibles) se aplicó el criterio Stepdown Bootstrap del procedimiento MULTEST del paquete de análisis estadístico SAS para el cálculo del valor de significación observada $\mathrm{P}$, de manera que el nivel de significación del conjunto de hipótesis estadísticas a probar fuese igual a 0,05 (a $=0,05)$. El criterio Stepdown Bootstrap determina el valor $\mathrm{P}$ mediante muestreos repetidos dentro de las observaciones para generar una población del estadístico de prueba t, tomando en consideración el número de comparaciones que se realiza simultáneamente.

\section{Resultados}

\section{Variables ambientales}

Se presentan los resultados de las variables climáticas registrados con los equipos de precipitación y temperatura (Figuras 4, 5, 6 y 7). 


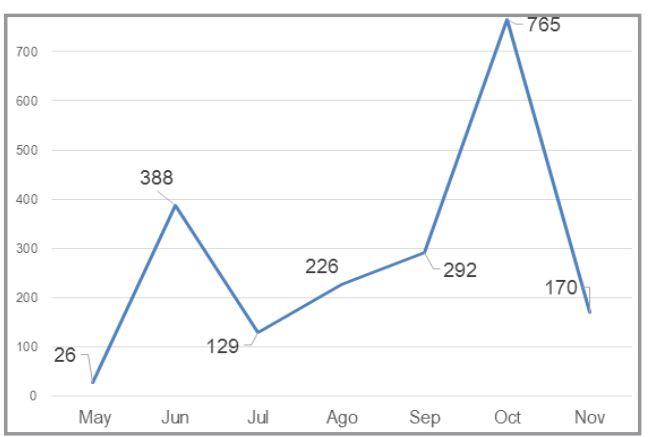

Figura 4. Precipitación mensual.

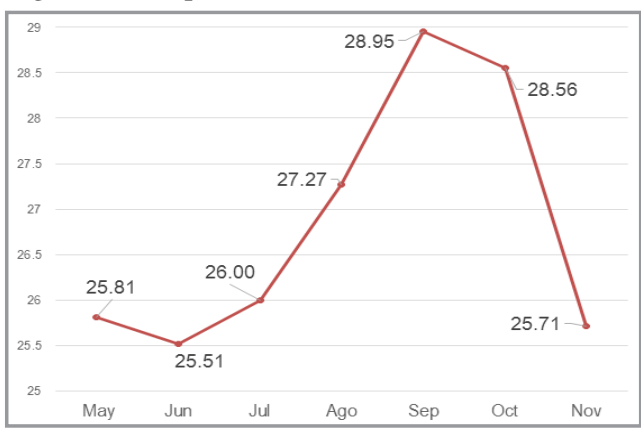

Figura 6. Temperatura máxima mensual.

La precipitación y temperaturas máximas $\left(\mathrm{T}_{\text {máx }}\right)$, mínimas $\left(\mathrm{T}_{\text {min }}\right)$ y medias mensuales fueron tomadas cada 29 minutos durante todo el período del experimento, desde el 18 de mayo hasta el 08 de noviembre del 2006. La precipitación total registrada fue de $1996 \mathrm{~mm}$ durante todo el periodo de toma de datos, la temperatura promedio fue de $15,7^{\circ} \mathrm{C}$, la $\mathrm{T}_{\text {máx }}$ $28,9^{\circ} \mathrm{C}$ en el mes septiembre y la $\mathrm{T}_{\text {mín. }} 4,7^{\circ} \mathrm{C}$ en el mes de julio. Un dato interesante de recalcar es que el día 21 de mayo del 2006 a las 22.59 horas se registró la primera gota de lluvia del estudio.

\section{Influencia del diámetro de tubo y agujeros de ventilación en el crecimiento inicial}

Para facilitar los análisis y simplificar las gráficas se han agrupado los tubos de la siguiente manera:

- Diámetro I que corresponde a tubos de 8,65 $\mathrm{cm}$ de diámetro (Diam.I)

- Diámetro II que corresponde a los tubos de 9,8 y 10,5 cm de diámetro (Diam.II)

- Diámetro III que corresponde a los tubos de

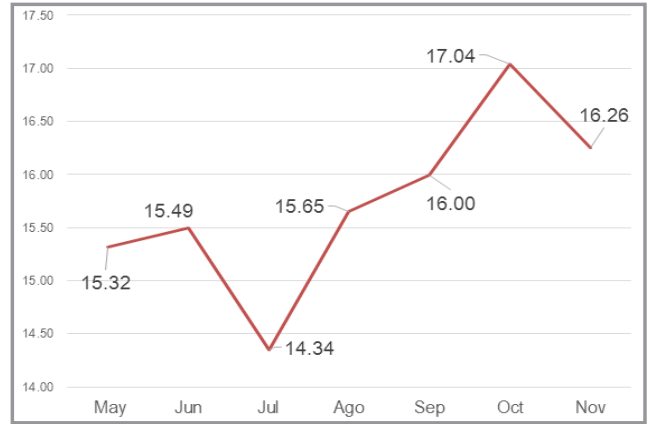

Figura 5. Temperatura media mensual.

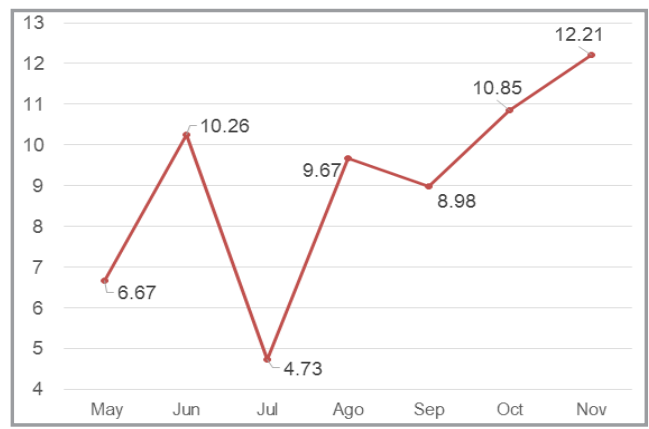

Figura 7. Temperatura mínima mensual.

11,5 y 12,15 cm de diámetro (Diam.III)

\section{Altura e incremento de altura por tratamiento}

Las Figuras 8 y 9 muestran los valores de altura (h) mensual y sus respectivos incrementos (Incr-h) según la clase diamétrica (Diam.I, Diam.II, Diam.III) y el grado de modificación ambiental inducido (tubo ventilado, no ventilado y testigo). Se incluyen además los Cuadros 2 y 3 que muestran los valores promedios de h y Incr-h en comparación con el testigo y su respectivo valor de significancia $P$.

\section{Diámetro e incremento de diámetro por trata- miento}

Las Figuras 10 y 11 muestran los valores de diámetro (d) mensual y sus respectivos incrementos (Incr-d) según la clase diamétrica (Diam.I, Diam.II, Diam.III) y el grado de modificación ambiental inducido (tubo ventilado, no ventilado y testigo). Se incluyen además los Cuadros 4 y 5 que muestran los valores promedios de d e Incr-d en comparación con el testigo y su respectivo valor de significancia $P$. 
Figura 8. Altura (h) mensual por tratamiento.

Figura 9. Incremento de altura (Incr-h) mensual por tratamiento.

Figura 10. Diámetro (d) mensual por tratamiento.

Figura 11. Incremento de diámetro (Incr-d) mensual por tratamiento.
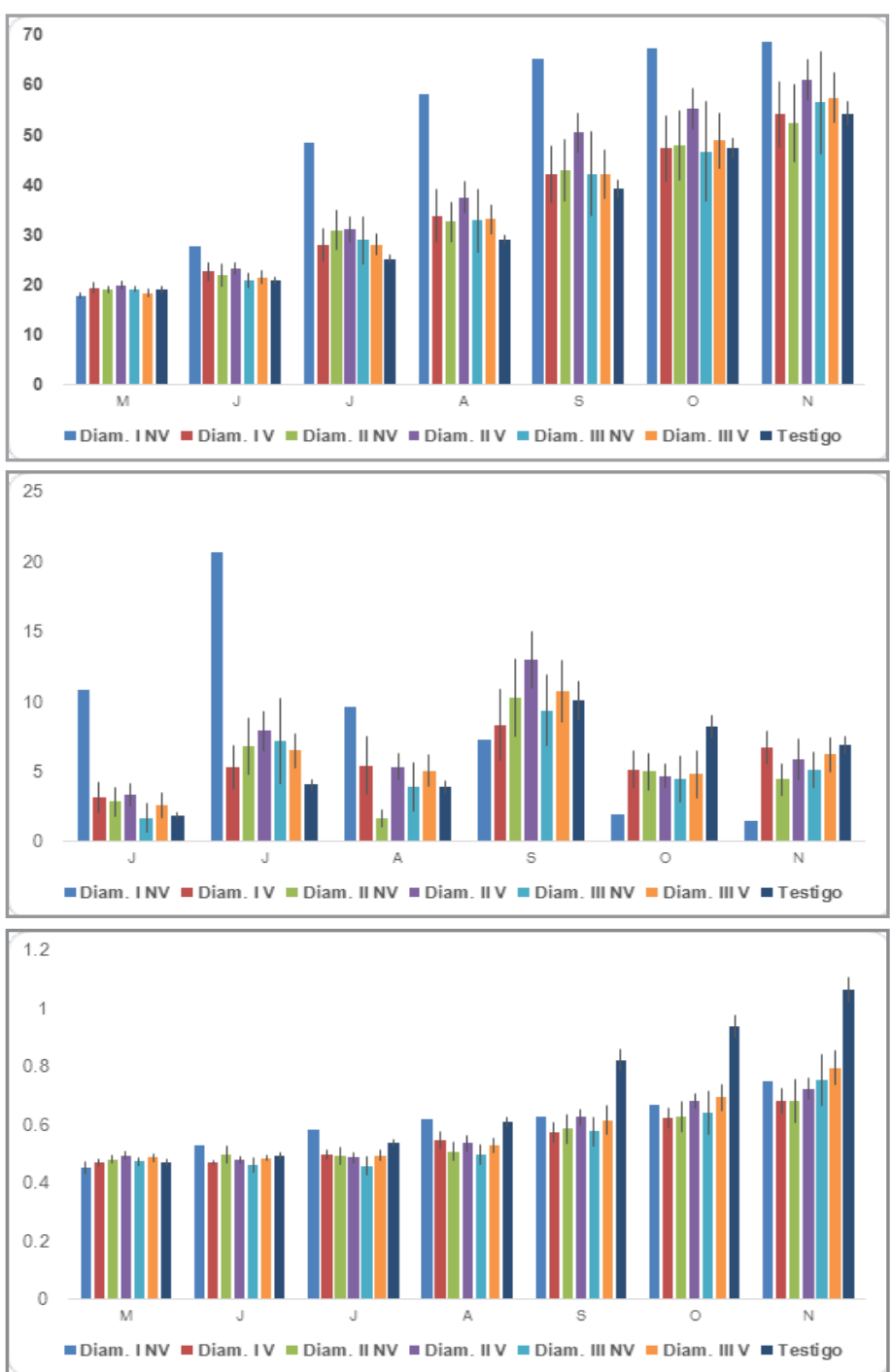

0.25

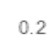

0.2

0.15

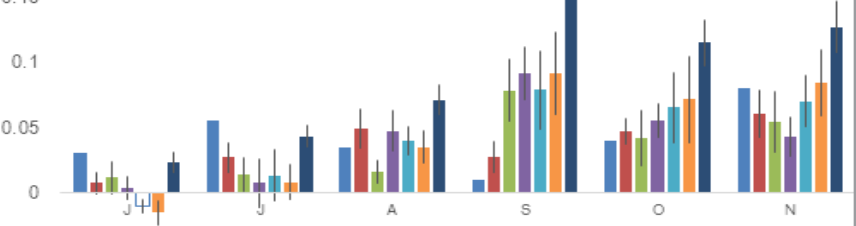

$-0.05$

-Diam. I NV = Diam. IV = Diam. II NV | Diam. II V = Diam. III NV = Diam. III V a Testigo 


\begin{tabular}{|c|c|c|c|}
\hline \multirow{2}{*}{ Mes } & \multicolumn{2}{|c|}{ Grupo } & $\begin{array}{c}\text { Valor P1 } \\
\text { (Stepdown } \\
\text { Bootstrap) }\end{array}$ \\
\cline { 2 - 3 } & Tratamiento & Testigo & 0,9219 \\
\hline Mayo & 18,94 & 19,05 & 0,2313 \\
\hline Junio & 22,98 & 20,86 & 0,0108 \\
\hline Julio & 32,55 & 25,15 & 0,013 \\
\hline Agosto & 37,98 & 29,05 & 0,0156 \\
\hline Septiembre & 47,54 & 39,23 & 0,1367 \\
\hline Octubre & 52,19 & 47,43 & 0,5670 \\
\hline Noviembre & 58,35 & 54,31 & 0,6161 \\
\hline
\end{tabular}

Cuadro 2. Comparación de valores $\mathrm{P}$ de promedios de $\mathrm{h}$ con tratamiento vs. testigo.

\section{Supervivencia por tratamiento}

La Figura 12 muestra los resultados de supervivencia los cuales indican diferencias extremas entre plantas con tubo protectores y testigos al inicio del estudio. Comparando solo plantas con tratamientos, (clase diamétrica y agujeros de ventilación) las clases de mayor diámetro con agujeros de ventilación ofrecen mayores posibilidades de supervivencia.

\section{Humedad del suelo}

La Figura 13 muestra los resultados de las mediciones de humedad de suelo cuyos valores son relativos y mayores a 9 en el inicio del estudio para todos los casos. Al no contar con bloques de yeso para cada individuo se repartieron 30 bloques entre los 5 diámetros de tubos con y sin ventilación, y los testigos de forma aleatoria en la parcela. Al término del estudio

\begin{tabular}{|c|c|c|c|}
\hline \multirow{2}{*}{ Mes } & \multicolumn{2}{|c|}{ Grupo } & $\begin{array}{c}\text { Valor P1 } \\
\text { (Stepdown } \\
\text { Bootstrap) }\end{array}$ \\
\cline { 2 - 3 } & Tratamiento & Testigo & 0,8497 \\
\hline Mayo & 0,477 & 0,469 & 0,8497 \\
\hline Junio & 0,488 & 0,492 & 0,831 \\
\hline Julio & 0,502 & 0,538 & 0,1331 \\
\hline Agosto & 0,540 & 0,609 & 0,0162 \\
\hline Setiembre & 0,602 & 0,823 & $<0,0001$ \\
\hline Octubre & 0,656 & 0,937 & $<0,0001$ \\
\hline Noviembre & 0,731 & 1,064 & $<0,0001$ \\
\hline
\end{tabular}

Cuadro 4. Comparación de valores $\mathrm{P}$ de promedios de $\mathrm{d}$ de tratamiento vs. testigo.

\begin{tabular}{|c|c|c|c|}
\hline \multirow{2}{*}{ Mes } & \multicolumn{2}{|c|}{ Grupo } & $\begin{array}{c}\text { Valor P1 } \\
\text { (Stepdown } \\
\text { Bootstrap) }\end{array}$ \\
\cline { 2 - 3 } & Tratamiento & Control & 0,0196 \\
\hline Junio & 4,07 & 1,86 & 0,0035 \\
\hline Julio & 9,06 & 4,06 & 0,06 \\
\hline Agosto & 5,18 & 3,90 & 0,3711 \\
\hline Setiembre & 9,84 & 10,06 & 0,9159 \\
\hline Octubre & 4,33 & 8,21 & 0,0087 \\
\hline Noviembre & 4,99 & 6,88 & 0,2594 \\
\hline
\end{tabular}

Cuadro 3. Comparación de valores $\mathrm{P}$ de promedios de Incr-h con tratamiento vs. testigo.

se observan valores relativos entre 8 y 9 en la mayoría de casos y un comportamiento similar de la humedad de suelo salvo algunas excepciones como es el caso del tubo de diámetro $12,5 \mathrm{~cm}$ no ventilado que terminó con un valor de 5,75. También se presentaron casos de pérdidas de bloque de yesos al final del estudio puesto que no se tienen datos registrados para algunos diámetros de tubo.

\section{Análisis foliar}

El Cuadro 6 muestra los resultados del análisis foliar realizado en el Laboratorio de Análisis de Agua, Suelo y Medio Ambiente (LAASMA) de la Universidad Nacional Agraria La Molina UNALM (Lima, Perú), la clave de campo indica el diámetro de tubo de menor a mayor valor, se tomaron cantidades similares de muestra foliar de tubos con y sin ventilación por cada diámetro distribuidos aleatoriamente en la parcela.

\begin{tabular}{|c|c|c|c|}
\hline \multirow{2}{*}{ Mes } & \multicolumn{2}{|c|}{ Grupo } & \multirow{2}{*}{$\begin{array}{c}\text { Valor P1 } \\
\text { (Stepdown } \\
\text { Bootstrap) }\end{array}$} \\
\hline & Tratamiento & Testigo & \\
\hline Junio & 0,005 & 0,023 & 0,0977 \\
\hline Julio & 0,021 & 0,043 & 0,1287 \\
\hline Agosto & 0,037 & 0,071 & 0,0526 \\
\hline Setiembre & 0,063 & 0,213 & $<0,0001$ \\
\hline Octubre & 0,053 & 0,114 & 0,0265 \\
\hline Noviembre & 0,065 & 0,127 & 0,0265 \\
\hline
\end{tabular}

Cuadro 5. Comparación de valores $\mathrm{P}$ de promedios de Inc.d de tratamiento vs. testigo. 


\begin{tabular}{|c|c|c|c|c|c|c|c|c|}
\hline $\begin{array}{c}\text { N. } \\
\text { Lab. }\end{array}$ & $\begin{array}{c}\text { Clave de } \\
\text { campo }\end{array}$ & $\begin{array}{c}\mathbf{N} \\
\mathbf{\%}\end{array}$ & $\begin{array}{c}\mathbf{P} \\
\mathbf{\%}\end{array}$ & $\begin{array}{c}\mathbf{K} \\
\mathbf{\%}\end{array}$ & $\begin{array}{c}\mathbf{C a} \\
\mathbf{\%}\end{array}$ & $\begin{array}{c}\mathbf{M g} \\
\mathbf{\%}\end{array}$ & $\begin{array}{c}\mathbf{S} \\
\mathbf{\%}\end{array}$ & $\begin{array}{c}\mathbf{N a} \\
\mathbf{\%}\end{array}$ \\
\hline 2532 & 1 & 2,29 & 0,19 & 2,31 & 1,39 & 0,38 & 0,14 & 0,02 \\
\hline 2533 & 2 & 2,85 & 0,27 & 2,97 & 2,09 & 0,51 & 0,12 & 0,02 \\
\hline 2534 & 3 & 2,24 & 0,31 & 3,37 & 1,48 & 0,51 & 0,17 & 0,02 \\
\hline 2535 & 4 & 2,82 & 0,22 & 2,64 & 1,65 & 0,41 & 0,12 & 0,02 \\
\hline 2536 & 5 & 2,52 & 0,23 & 3,24 & 1,72 & 0,42 & 0,14 & 0,02 \\
\hline 2537 & Testigo & 2,24 & 0,17 & 1,64 & 1,00 & 0,28 & 0,11 & 0,02 \\
\hline
\end{tabular}

Cuadro 6. Análisis foliar.

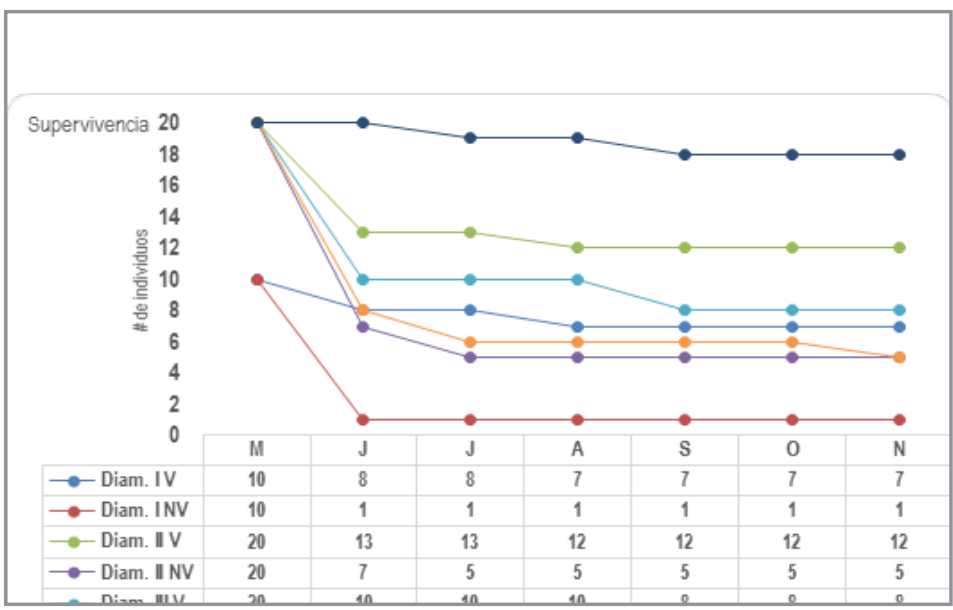

Figura 12. Supervivencia según tipo de tratamiento y testigo.

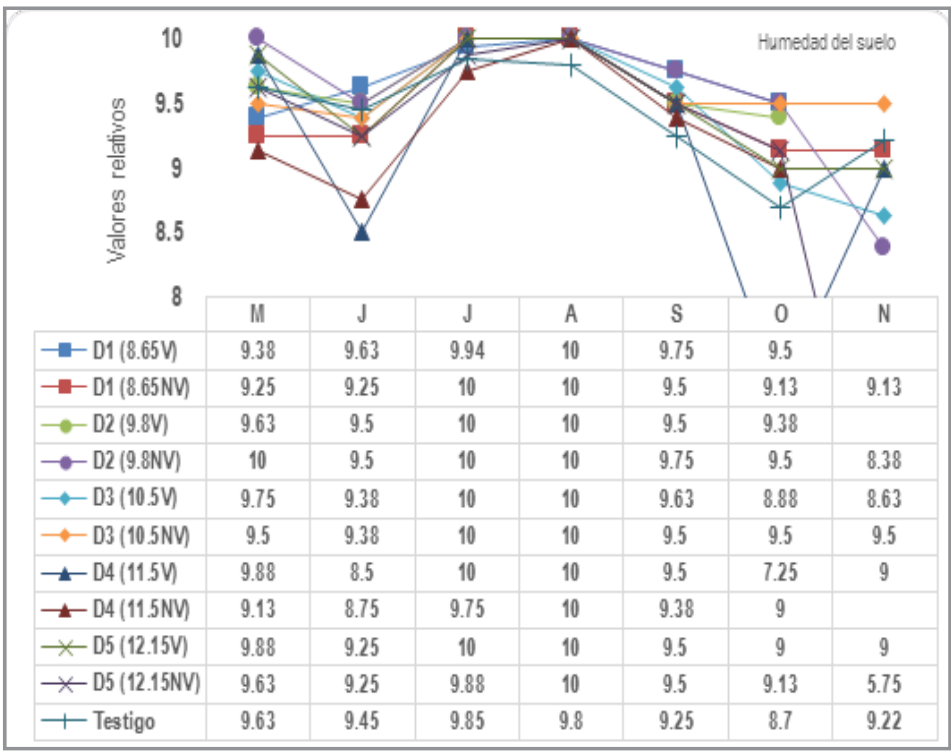

Figura 13. Datos relativos de humedad del suelo. 


\section{Discusión}

\section{Altura e incrementos de altura por tratamiento}

En la Figura 8 se observan los valores mensuales de altura el cual es mayor en las plantas con tratamiento que los testigos en casi todos los casos. De acuerdo a Potter (1991) este es el efecto más común de los tubos protectores en el crecimiento inicial de especies forestales. Comparando solo individuos con tratamiento, el diámetro I con el tubo no ventilado (Diam.I$\mathrm{NV}$ ) muestra valores mayores de altura en casi todos los meses sin embargo su supervivencia es la más baja ya que estos valores corresponden a un solo individuo que tuvo un notable desarrollo. Esto indica que se deben tener en cuenta factores como las condiciones del sitio y calidad de planta antes de determinar el uso de protectores (Potter 1991). La Figura 9 muestra un incremento mucho mayor en los primeros meses para el tubo con menor diámetro no ventilado pero como se trata de un solo individuo con un muy buen crecimiento, el motivo de este desarrollo puede ser más por aspectos fisiológicos (calidad de planta) que por los tratamientos, no hay la suficiente información para determinar si esto es inducido por el uso del tubo protector. En el mes de octubre, en el cual se registró la mayor precipitación mensual con $765 \mathrm{~mm}$, los incrementos de altura se reducen considerablemente para todos los tratamientos, el testigo disminuye ligeramente a comparación de los tratamientos en septiembre, esto puede deberse a una acumulación de agua en los tubos o a la generación de condiciones idóneas para el desarrollo de fitopatógenos que pueden afectar el crecimiento del individuo (West et al. 1999). El análisis estadístico entre plantas con tratamiento no genera diferencias estadísticas significativas entre los valores de altura (prueba de $t$, método Stepdown Bootstrap, $\mathrm{P}>0,05$, resultados no mostrados). Sin embargo cuando se compara mediante la prueba de $t$ el promedio mensual de altura de los distintos grupos de tratamientos con el grupo testigo se observaron diferencias estadísticamente significativas en los meses julio y agosto $(\mathrm{P}<0,05)$ (Cuadro 2). El Cuadro 3 presenta la misma situación para el análisis estadístico de incrementos de altura, las diferencias significativas se dieron en los meses de junio, julio y octubre comparando plantas con tratamiento vs. testigos.

\section{Diámetro e incrementos de diámetro por tratamiento}

Los valores mensuales de diámetro muestran una similar tendencia para todos los individuos con tratamiento, entre estos el que mostró mejores resultados fue el Diam.III-V aunque la diferencia es mínima (Figura 10). En el estudio realizado en el periodo 1993-1995 en Estados Unidos con 11 especies forestales West et al. (1999) indica que los tubos protectores no tuvieron efecto significativo alguno en el desarrollo diametral del árbol. Potter (1991) indica lo mismo sobre el desarrollo diametral de plantones sin embargo resalta que puede haber un efecto en la estabilidad del tallo por su menor crecimiento dependiendo del tiempo de permanencia del tubo protector que le da estabilidad a la planta. El tratamiento testigo muestra valores más elevados, esta diferencia aumenta a partir del mes de septiembre con el aumento de la precipitación, esto se observa de forma clara en la Figura 11. Al igual que en el punto anterior no se observaron diferencias estadísticas significativas entre los promedios de diámetro e incremento de diámetro entre los grupos experimentales (prueba de $t$, método Stepdown Bootstrap, $\mathrm{P}>0,05$, resultados no mostrados). Comparando los resultados con los testigos se tienen diferencias estadísticamente significativas en los meses agosto, setiembre, octubre y noviembre $(\mathrm{P}<0,05$, Cuadro 4$)$ para los valores mensuales de diámetro y en los meses de septiembre, octubre y noviembre $(\mathrm{P}<0,05$, Cuadro 5) para la variable incremento de diámetro.

\section{Supervivencia por tratamiento}

El tratamiento testigo es el que muestra un mejor comportamiento, con un $90 \%$ de supervivencia, al final del experimento. La prueba de independencia chi cuadrado indica que la supervivencia no es independiente del tratamiento. Comparando los tratamientos, el grupo Diam.II-V terminó con 12 plantas vivas al (60\% supervivencia). El grupo Diam.I-V ter- 
minó con un $70 \%$ supervivencia sin embargo el número de individuos fue de 10 plantones a diferencia de los grupos II, III y testigo con 20 individuos. El grupo Diam.I-NV terminó con 1 individuo de un total de 10 plantas siendo el más bajo de todos. Potter (1991) indica que en la mayoría de experimentos realizados con tubos protectores la supervivencia fue al 100\% a diferencia del $75 \%$ en plantas testigo. Sin embargo indica algunas excepciones con mayores tasas de supervivencia de testigos atribuyendo como posibles causas los cambios bruscos de temperatura en el interior del tubo protector, la calidad de la planta y un inadecuado control de malezas. Es importante resaltar que los valores de supervivencia cambiaron bruscamente durante el primer mes del estudio, a partir junio la tasa de supervivencia se mantuvo estable con ligeras variaciones.

\section{Humedad del suelo}

La Figura 13 muestra un comportamiento similar de humedad del suelo para todos los tratamientos incluyendo el testigo. Este dato es importante para propósitos de irrigación en condiciones controladas (Delmhorst 1999), por su parte Potter (1991) indica que los principales factores que caracterizan el microclima de los tubos protectores son elevadas temperaturas, alta humedad, menor movimiento de aire y reducida intensidad de luz. La complicada interacción de estos factores dificulta el análisis del efecto del tubo protector en el aporte de agua y por ende el desarrollo de la planta, sin embargo menciona que dentro de estos parámetros hay algunos que están más ligados a indicadores como crecimiento inicial o supervivencia. Finalmente recomienda el uso del indicador Déficit de Presión de Vapor (DPV) como más apropiado y el cual refleja con mayor exactitud la influencia de la humedad atmosférica en los procesos fisiológicos de las plantas en condiciones microclimáticas inducidas como en el tubo protector.

\section{Análisis foliar}

El análisis muestra que todos los tratamientos con tubos tienen mayores concentraciones de nutrientes, esto puede ser debido a que en los individuos testigos hay una mayor dilución de los nutrientes con el aumento del crecimiento $\mathrm{y}$ en los tubos se mantiene una concentración alta debido a sus paredes que mantiene el suelo unido reduciendo su pérdida y por ende facilitando su aprovechamiento. No se ha encontrado información relevante sobre este punto.

\section{Conclusiones}

El tratamiento (clase diamétrica y ventilación) favoreció el crecimiento en altura en comparación con los individuos testigos pero sucede lo contrario en cuanto al crecimiento diametral. Esta diferencia se debe al efecto de etiolación debido a la falta de luz dentro del tubo protector lo que hace que el plantón desarrolle más su crecimiento en altura. Comparando solo plantas con tubos protectores, el tubo ventilado dio mejores resultados que los no ventilados.

Los resultados obtenidos por tratamiento no son significativos debido a la alta mortalidad obtenida, llegando a medirse 1 solo individuo en el caso del grupo Diam.I-NV, que corresponde al grupo con mejores valores de crecimiento en altura alcanzada de acuerdo a los resultados. Es necesario realizar estudios con mayor cantidad de individuos y menor número de variables para obtener resultados más confiables en próximos estudios.

En cuanto a la supervivencia sobresale el grupo experimental Diam.II-V con un $60 \%$ de plantones vivos sin embargo es menor al testigo con un $90 \%$. Para determinar datos certeros sobre este parámetro es necesario realizar estudios más detallados incluyendo variables microclimáticas modificadas en el tubo protector, procesos fisiológicos en las plantas, calidad de planta y contrastarlo con las condiciones del sitio de estudio.

La humedad del suelo de los plantones con tubos protectores resultó ligeramente superior a los testigos, esto es un indicador positivo del efecto de condensación de neblinas sin embargo no es suficiente para determinar el uso de tubos protectores en un programa de 
reforestación durante periodos de baja pluviometría.

A través del análisis foliar se observa una influencia positiva en el desarrollo fisiológico de las plantas con tubos, por su mayor porcentaje de nutrientes que las plantas testigo. Es necesario realizar mayores estudios en diversas condiciones de sitio y compararlo con evaluaciones relacionadas a la calidad de planta para determinar con mayor exactitud el aporte de los tubos protectores en este aspecto.

\section{Agradecimientos}

$\mathrm{Al}$ Sr. Otto Bottger por el generoso ofrecimiento de su Fundo "Ranchería" para el trabajo de campo y al Sr. Miguel Bottger (QEPD), por su gran hospitalidad en cada visita de evaluación. Al Dr. Damien Catchpole, por su importante soporte técnico en la instalación del sensor para los registros de temperatura y precipitación en la parcela. Al Sr. Luis Fernando Rego de TUBEX, UK por la donación de los Tree shelters usados en este experimento. Al Dr. Percy Summers del Proyecto Pro Pachitea del IBC por las facilidades logísticas y el apoyo económico brindado. Al Dr. David Ramírez por el envío de material bibliográfico y por sus enseñanzas, orientaciones y sugerencias relacionadas a temas ecofisiológicos y experimentales.

\section{Bibliografía}

Acosta, B. 1996. Las precipitaciones ocultas y sus aplicaciones a la agricultura. Ornex, FR. FAO, CTA, JRC-EU, OMM, AAA, S.A.R.L. 170 p.

Bergez, JE; Drupaz, C. 2000. Effect of ventilation on growth of Prunus avium seedlings grown in treeshelters. Agricultural and Forest Meteorology 104: 199 - 214.

Catchpole, D. 2012. Orographic gradients in climate and forest cover at the Cordillera Yanachaga, Peru. Tesis PhD. Tasmania, AU. University of Tasmania. $268 \mathrm{p}$.

Del Campo, AD; Aguilella, A; Gonzalez E; Navarro, RM. 2006. Effect of tree shelter design on water condensation and run-off and its potential benefit for reforestation establishment in semiarid climates. Forest Ecology and Management 235 (1-3): 107 - 115.

DELMHORST, 1999. Soil moisture measuring system. Model KS-D1. Owner's manual. www. delmhost.com. 16 p.

Filomeno, L. 2008. Vivero Forestal (entrevista). Lima, PE, Coordinador Nacional Fondebosque Pucallpa.

Gutierrez, A. 2007. Efecto del control de malezas sobre la disponibilidad de Agua en el suelo y en las variables de crecimiento en plantaciones de Pinus radiata D. Don, de cuarenta y cuatro meses, en sectores de secano y costa (VII región). Tesis Ingeniero Forestal. Santiago, Chile. 48p.

Kjelgren, R; Rupp, L. 1997. Establishment in tree shelters I: Shelters reduce growth, water use, and hardiness, but not drought avoidance. Hortscience 37(7): 1281-1283.

Kjelgren, R; Montague, D; Rupp, L. 1997. Establishment in tree shelters II: Effect of shelter color on gas exchange and hardiness. Hortscience 37(7): 1281-1283.

Marzol, M. 2005. La captación de agua de la niebla en la isla de Tenerife, Caja General de Ahorros de Canarias. 220 p. (Publicación $\mathrm{N}^{\circ}$ 333, Investigación $\mathrm{N}^{\circ}$ 61).

Pearcy, RW; Ehleringer, J; Mooney, HA; Rundel, PW. (Eds.) 1994. Plant Physiological Ecology: Field Methods and Instrumentation. Londres, GB. Chapman \& Hall. 441p.

Potter, MJ. 1991. Tree shelters. Londres, GB. HMSO, 48 p. (Forestry Commission Handbook No7).

Reynel, C.; Pennington, TD; Pennington, RT; Marcelo, JL; Daza, A. 2006. Árboles útiles del Ande Peruano: una guía de identificación, ecología y propagación de las especies de la Sierra y los Bosques Montanos del Perú. Lima, PE. Darwin Initiative, Project 332; Royal Botanic Garden, Edinburgh; UNALM, Royal botanic Garden, Kew; APRODES. 466p.

TUBEX, UK. 2006. Market leaders in plant care solutions (en línea). Consultado mayo 2006. Disponible en http://www.tubex.com 
TUBEX, UK. 2007. Standard Range. Tubex data sheets, installation and case studies. Disponible en http://www.tubex.com.

West, H; Chappelka, H; Tilt, K; Ponder, H; Williams, J. 1999. Effect of tree shelters on survival, growth, and wood quality of 11 tree species commonly planted in the southern United States. Journal of Arboriculture 25(2): 69-75. 